\title{
Regaining quantum incoherence for matter fields
}

\author{
Pedro F. González-Díaz \\ Instituto de Optica, Consejo Superior de Investigaciones Cientificas, Serrano 121, 28006 Madrid, Spain
}

(Received 8 July 1991)

\begin{abstract}
The possible quantum state of wormholes or little baby universes should be described by a nonfactorizable density matrix given by the path integral over the class of asymptotically flat four-geometries and asymptotically vanishing matter-field configurations which suitably match the prescribed data on threesurfaces which do not divide the manifold on the inner boundary. An instanton is here obtained which can represent such a nonsimply connected wormhole manifold, and is used to evaluate the asymptotic effective interaction of the resulting correlated baby universes with ordinary quantum fields at low energies in the Fock representation. It is argued that the demand of locality on the interacting quantum field commutators is no longer valid for correlated baby universes, and it is therefore concluded that quantum coherence in the matter-field sector is lost as a consequence of the interaction with nonsimply connected wormholes. A proposal is advanced that wormholes may provide us with a complementary quantum state sector that would induce the collapse of the state vector in the quantum measurement of any observable for ordinary microscopic matter systems.
\end{abstract}

PACS number(s): 04.60. $+\mathrm{n}$, 03.65.Bz, 04.20.Jb

\section{INTRODUCTION}

In the last fifteen years or so there has been some debate concerning whether quantum gravity will induce a breakdown of predictability in the underlying quantum description of ordinary matter fields [1-6,9-11]. The subject is of the utmost interest both in physics and philosophy, for, if eventually the controversy were finally decided in favor of a corresponding loss of quantum coherence, then it is currently believed that our physical theories could not predict anything, or, in other words, there would not be any possible actual representation of the physical world.

To make the issue clear enough from the beginning, one should state all at once what exactly is meant by a loss of quantum coherence in the present context. Essentially, it simply describes the evolution of an initially pure state in the ordinary matter-field sector into a mixed state, this change being induced exclusively by the effective interaction of the matter fields with some otherwise inobservable nontrivial gravitational instantons. It is worth noticing that this kind of interaction has nothing to do with any conventional interaction between matter fields, or nonlinear quantum-optical causes.

The idea was first advanced by Hawking [1] who realized that in non-Minkowskian spacetimes any correlation between two given systems is irretrievably lost when one of them gets beyond the horizon of the other. The implications of this idea prompted vigorous opposition, mainly on the part of Gross who argued [3] that this would ultimately lead to a breakdown of unitary evolution in quantum mechanics. More recently, however, Hawking presented new arguments for the loss of quantum coherence by using wormholes $[4,5]$, rather than simply connected spacetimes. Quite at the same time, Lavrelashvili, Rubakov, and Tinyakov [6], made a similar proposal.

A wormhole is usually given as an instanton and represents a tunneling between an initial state which is just flat space, and a final state representing flat space plus a baby universe (typically a very little closed universe) which branches off from the asymptotic flat region. This process may only occur in Euclidean gravity, and gives rise to a change in the topology [7]. Throughout this paper, we will be referring to baby universes which connect two otherwise disconnected large flat regions of spacetime. A wormhole end will always insert only at one point in the large regions, but the wormhole manifold itself may have any topology. When it is in a pure state, representable by a wave function [4], the wormhole topology has to be simply connected; if, in turn, it is in a mixed state describable only by a density matrix [8], the wormhole topology will be multiply connected.

However, once again and apparently in quite a definitive way this time, Coleman and Giddings and Strominger [9], and others [10] argued that the production of baby universes does not lead to any loss of quantum coherence in the matter-field sector. By demanding locality in the parent universe, Coleman was able to show that, when performing a large number of experiments, the initial family wave function collapses into a certain state which remains invariant throughout the interaction with baby universes. Hawking and Laflamme [11] elaborated on this a little bit further.

Unruh has recently stressed [12] that if we identified two baby universes connected to the large region by a wormhole instanton, then the resulting spacetime still is a solution to the classical equations. Thus, such a solution connected two different points in the four-geometry by a handle on the large spacetime and led to a highly nonlocal effective action, violently unbounded from below, which, in addition to showing a loss of quantum coherence, destroyed the Euclidean quantum theory. (The instability would be much more severe than the usual con- 
formal instability of the quantized Euclidean action [13].) In this work, we shall show that the nonlocal effects which are responsible for the loss of quantum coherence ought to be related to the contribution of wormholes with multiply connected topologies, which allow no further instabilities in the Euclidean quantum theory, rather than the kind of processes considered by Unruh.

The outline of the work is as follows. In Sec. II we discuss the quantum state of wormholes. It is argued that such a state must be given by a nonfactorizable density matrix, rather than a proper wave function. We discuss also a Euclidean solution for the case of two massless scalar fields coupled to Hilbert-Einstein gravity, which can represent a doubly connected wormhole manifold. Section III deals with the effective vertex corresponding to the interaction of a scalar matter field with baby universes which are produced or destroyed, two at a time. An approximate expression for the baby-universe interaction operator is obtained. A discussion on the locality requirements is given in Sec. IV. It is shown that the demand of locality can no longer hold on operators of the two sectors separately when the wormhole is doubly connected. Using counterarguments to Coleman's we show then that wormholes induce an irretrievable loss of quantum coherence in the matter sector of the theory. Finally, a brief discussion of the results is included in Sec. V. This section also includes the new suggestion that wormholes may provide a complementary quantum-state gravitational sector which is responsible for the reduction of the quantum-state vector of ordinary matter during its nonunitary evolution induced by measurement.

Throughout this work, we shall use units so that $\hbar=c=G=1$.

\section{THE STATISTICAL STATE OF WORMHOLES}

The quantum state of wormholes has been described [4] by a wave function which is a functional of the metric and matter-field configurations on a given three-sphere $S$ which divides the four-manifold $M$ into two disconnected parts $\boldsymbol{M}_{+}$and $\boldsymbol{M}_{-}$. In this case, the probability for a given three-geometry and matter-field configuration, $P\left[h_{i j}, \phi_{0}\right.$, factorizes into two wave functions

$$
P\left[h_{i j}, \phi_{0}\right]=\Psi_{+}\left[h_{i j,} \phi_{0}\right] \Psi_{-}\left[h_{i j}, \phi_{0}\right],
$$

where $\Psi_{+}$and $\Psi_{-}$are given by the path integral over all metrics and matter fields on $M_{+}$and $M_{-}$, respectively. If the matter fields involved are $C P$ invariant, then one has a proper wave function

$$
\begin{aligned}
\Psi\left[h_{i j}, \phi_{0}\right]=\Psi_{+}\left[h_{i j}, \phi_{0}\right] & =\Psi_{-}\left[h_{i j}, \phi_{0}\right] \\
& =N \int_{C} d g_{\mu \nu} d \phi e^{-I\left[g_{\mu v}, \phi\right]},
\end{aligned}
$$

where $N$ is a normalization factor and $I$ denotes Euclidean action.

The path integral in (2.2) is over the class $C$ of Euclidean four-geometries which are asymptotically flat and have an inner boundary $S$ with topology $S^{3}$ and metric $h_{i j}$, and over matter-field configurations which are zero at infinity and match the given values of $\phi_{0}$ on $S$. Thus, the wormhole would be in a pure quantum state and the topology of manifold $M$ is simply connected. (We consider the asymptotically flat regions connected by the wormhole tube to be otherwise disconnected from each other.)

More recently, it has been proposed [8] however, that the most general probability $P\left[h_{i j}, \phi_{0}\right]$ for wormholes should not generally factorize, so that the quantum state of wormholes would be represented by a density matrix $\rho$, rather than a wave function $\Psi$. Then, the wormhole would be in a mixed state. The proposal is made precise by giving the path integral

$$
\rho=N^{2} \int_{C} d g_{\mu \nu} d \phi e^{-I\left[g_{\mu \nu}, \phi\right]},
$$

where the integral is over the class $C$ of asymptotically flat four-geometries and asymptotically vanishing matter-field configurations which match the prescribed data $\left[h_{i j}, \phi_{0}\right]$ on its three-surface $S$, and the orientation reverse of $\left[h_{i j}^{\prime}, \phi_{0}^{\prime}\right]$ on its three-manifold $S^{\prime}, S$ and $S^{\prime}$ together forming the boundary of each four-geometry in the path integral. Thus, (2.3) should be interpreted as describing the actual statistical quantum state of a single wormhole.

The mixed state given by (2.3) will correspond to a wormhole manifold $M$ which is nonsimply connected. One of such manifolds can be represented by an instanton obtained by simultaneously coupling a minimal massless scalar field $\varphi$ and a conformally invariant massless scalar field $\phi$ to Hilbert-Einstein gravity. For a RobertsonWalker minisuperspace, the resulting Euclidean action is

$\widetilde{I}=\frac{1}{2} \int d \eta N\left[a^{2} \frac{\varphi^{\prime 2}}{N^{2}}+\frac{\chi^{\prime 2}}{N^{2}}+\chi^{2}-\frac{a^{\prime 2}}{N^{2}}-a^{2}\right]=\int d \eta N L$,

where $\eta=\int d \tau / a$, a prime denotes $d / d \eta, a$ is the scalar factor, $N$ is the lapse function, and $\chi=a \phi$. From the equations of motion for $a, \varphi$, and $\chi$, we obtain (in the gauge $N=1$ )

$$
\begin{aligned}
& a^{2} \varphi^{\prime}=\frac{1}{2} K, \quad \chi^{2}-\chi^{\prime 2}=R_{0}^{2}, \\
& a^{\prime \prime}=a-\frac{K^{2}}{4 a^{3}}-f(a, K),
\end{aligned}
$$

with $K$ and $R_{0}$ being integration constants.

The solution to (2.5) and (2.6) and the Hamiltonian constraint are

$$
a(\eta)_{ \pm}=\left(\frac{1}{2}\right)^{1 / 2}\left[R_{0}^{2} \pm\left(R_{0}^{4}-K^{2}\right)^{1 / 2} \cosh 2 \eta\right]^{1 / 2} .
$$

Solutions $a_{+}$and $a_{-}$lie in the two disconnected, classically allowed regions for which the potential

$$
V=\frac{1}{2}\left(a^{2}+\frac{K^{2}}{4 a^{2}}-R_{0}^{2}\right)
$$

is negative. It is only $a_{+}$which can be thought of as giving the metric of a wormhole, $a_{-}$is a singular solution confined to be $\leq a_{-}(0)$, and hence the isotropic manifold provided with a metric given by $a_{-}$is disconnected from 
the wormhole manifold and thereby from the two asymptotically flat regions, for any given allowed values of $R_{0}^{2}$ and $K$. For $K=0, a_{+}=R_{0} \cosh \eta$ and $a_{-}=i R_{0} \sinh \eta$, so that there is no real disconnected region, and the two asymptotically flat regions are simply connected to each other [7]. However, for $K \neq 0$, the values taken by $a_{-}$along the conformal time interval $\left(0, \eta_{0}\right.$ $\left.=\operatorname{arccosh}\left[\left(1-K^{2} / R_{0}^{4}\right)^{-1 / 2}\right]\right)$ are nonzero and real, inducing the appearance of a real nonzero disconnected region inside the wormhole manifold. Hence, the wormhole manifold will be doubly connected for $K \neq 0$. (See Fig. 1 for a two-dimensional pictorial representation of that manifold.) More complicated multiply connected wormhole topologies can be obtained, e.g., by using gravity theories containing higher-derivative terms [14].

Baby universes are leaked off at the two necks around the minima $\pm(K / 2)^{1 / 2}$ as solutions of the corresponding Lorentzian equations of motion. Such solutions represent tunneling [15] between the two otherwise disconnected, classically allowed regions described by the Euclidean solutions.

Throughout this paper we shall use this instanton to represent a doubly connected wormhole manifold. Concerning such a manifold, a point is worth emphasizing. The two inner three-manifolds at $\eta=0$ are correlated. In fact, it can be seen that the single-time covariance of fluctuations of the scale factor at the two equilibria points obtained from potential $V$ is unity [16]. This can be interpreted by considering that, since wormholes are spacetime fluctuations, if the correlation between the two disjoint three-manifolds disappeared, it took with it the doubly connectedness of the manifold, leaving it simply connected. Now, the existence of this coherence effect should induce a decrease of the entropy for the whole sys-

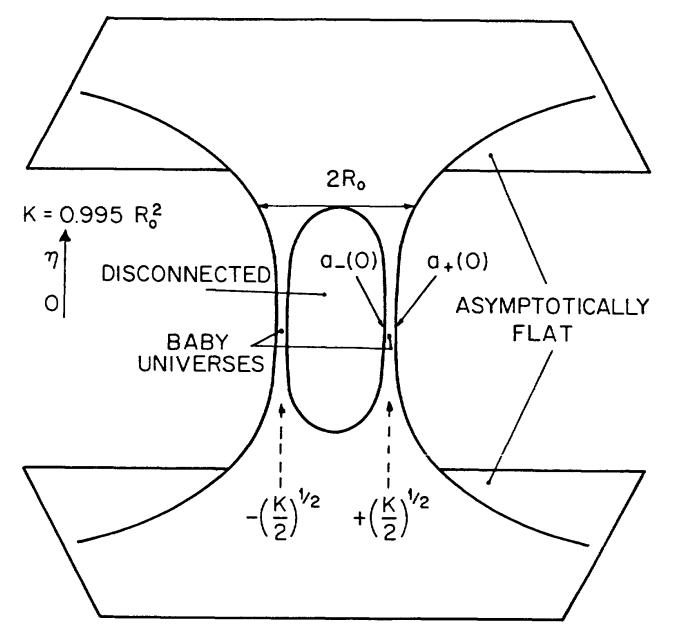

FIG. 1. Schematic representation of a doubly connected wormhole. Values of $R_{0}$ and $K$ are very close to each other, but still satisfying $R_{0}^{2}>K$. At the limit $K \rightarrow R_{0}^{2}$ no baby universe can be produced, and the two large regions become disconnected. tem. This means that some information about the babyuniverse system as a whole became available to observers in the asymptotic regions. Or in other words, baby universes would no longer be disconnected from the asymptotic regions and, therefore, the two disjoint inner three-manifolds could no longer divide the four-manifold into two parts. Thus, the state of the wormhole configuration pictured in Fig. 1 must be given by a nonfactorizable density matrix rather than a wave function.

The existence of correlations on the inner threemanifold leads to a topological consequence of interest to the issue of a possible loss of quantum coherence. It follows that, whereas for simply connected wormholes, the insertion point $x_{0}$ on the flat region maps to a unique point along the entire four-manifold, for a doubly connected wormhole, the end point $x_{0}$ on the flat region is mapped to a pair of spacelike separated points on the inner three-manifold, the proper separation distance being $(2 K)^{1 / 2}$.

\section{THE BABY-UNIVERSE INTERACTION OPERATOR}

The quantum state of a wormhole given by a wave function is certainly an incomplete state. Because an observer in the asymptotic region does not know anything about the values of $h_{i j}$ and $\phi_{0}$ on the three-surface $S$, in order for defining an actual quantum state of wormholes one should integrate the path integral (2.1) over $h_{i j}$ and $\phi_{0}$. This would leave a quantum state given by a functional with no argument, or in other words, a probability for nothing. However, the state given by a density matrix (2.3) will make a suitable quantum state for wormholes as, in this case, there always exists an observational connection between the baby-universe space-time sector and the main large nearly flat regions induced by the abovediscussed correlations between the two inner threemanifolds. In any event, the states given both by (2.1) and (2.3) have still a clear operational meaning: Instead of worrying about the quantum state for an isolated noninteracting wormhole, one is usually rather interested in calculating the effects of wormholes on ordinary matter at low energies by using either a factorized Green's function

$$
\left\langle 0\left|\phi\left(x_{1}\right) \cdots \phi\left(x_{r}\right)\right| \Psi\right\rangle
$$

in the case of simply connected wormholes [4], or a full Green's function

$$
\left\langle 0\left|\phi\left(x_{1}\right) \cdots \phi\left(x_{r}\right)\right| \rho\left|\phi\left(y_{1}\right) \cdots \phi\left(y_{r}\right)\right| 0\right\rangle,
$$

for multiply connected wormholes [8] (here, typically, the $\phi$ 's are conformally invariant scalar particles) which is given by a path integral where the required integrations have been included. For the second case, the propagator can be written 


$$
\int d\left[h_{i j}\right] d\left[\phi_{0}\right] d\left[h_{i j}^{\prime}\right] d\left[\phi_{0}^{\prime}\right] \rho \int_{C} d\left[g_{\mu \nu}\right] d[\phi] \phi\left(x_{1}\right) \cdots \phi\left(x_{r}\right) \phi\left(y_{1}\right) \cdots \phi\left(y_{r}\right) e^{-I\left[g_{\mu \nu}, \phi\right]}
$$

The Green's function (3.1) was evaluated in Ref. [8] and produced bilocal interactions proportional to

$$
\int \frac{d^{4} x_{0}}{\left(x_{1}-x_{0}\right)^{2}\left(x_{2}-x_{0}\right)^{2}} \int \frac{d^{4} x_{0}^{\prime}}{\left(y_{1}-x_{0}^{\prime}\right)^{2}\left(y_{2}-x_{0}^{\prime}\right)^{2}},
$$

just as for the case in which we use a wave function [4].

The integrations over the positions $x_{0}$ and $x_{0}^{\prime}$ in the two asymptotic regions should ensure conservation of energy on both flat regions. Although this will be rigorously true for wave functions $\Psi$ (because in this case baby universes are closed universes with zero energy and momentum), for the case of the density matrix $\rho$, energy conservation applies to the joint system formed by the flat regions plus the baby universes, but not necessarily to either of the two subsystems separately. One should expect that, as in the case of the density matrix, the total energy and momentum of the baby universes are no longer zero. Here, the wormhole need not be in shell in the sense that the Wheeler-DeWitt operator acting on $\rho$ is no longer zero [8]. (For instance, for the wormhole manifold considered in Sec. III, the total energy of the involved baby universes ought to be proportional to parameter $K$.) Hence, the Gell-Mann-Low formula, which is perfectly applicable in the case of simply connected wormholes and leads to an effective interaction Hamiltonian term

$$
\sum_{i} H_{i}(\phi)\left(a_{i}^{\dagger}+a_{i}\right)
$$

(where the discrete index $i$ collectively labels the types of baby universes, the $H_{i}$ 's are matter-field interaction Hamiltonian terms, and the $a_{i}$ 's are Fock operators for the baby universes) can no longer be used for nonsimply connected wormholes.

The baby-universe interaction operators $A_{i}{ }^{\mathrm{DC}}$ for a doubly connected wormhole, which played the role of the operator $A_{i}^{\mathrm{SC}}=a_{i}^{\dagger}+a_{i}$ in (3.3) will be obtained here using a combinatorics which parallels that used by Coleman [9], unless for the distinguishing feature that now we have two correlated identical baby universes per wormhole end. This analysis leads to a sum over doubly connected wormholes given by

$$
\Sigma e^{-S}=\sum_{r=0}^{\min \left(n_{1}, n_{2}\right)} \frac{e^{\beta^{2} / 2}\left[\left(2 n_{1}\right) !\left(2 n_{2}\right) !\right]^{1 / 2} \beta^{n_{1}+n_{2}-2 r}}{\left(n_{1}-r\right) !\left(n_{2}-r\right) !(2 r) !}
$$

where $n_{1}$ and $n_{2}$ denote the number of baby-universe pairs in the initial and final state, respectively, and

$$
\beta=C e^{-2 S_{0}} V_{4} \text {, }
$$

in which $C$ is some constant, $S_{0}$ is the action of a semiwormhole, and [9] $V_{4}=\int_{M} d^{4} x g^{1 / 2}$.

Because two baby universes are simultaneously in- volved in the simplest interaction process for doubly connected wormholes, one cannot have an exact expression for the baby-universe interaction operator. The BakerCampbell-Hausdorff formula for two operators $O_{1}$ and $O_{2}, e^{O_{1}} e^{O_{2}}=e^{O_{1}+O_{2}} e^{\left[O_{1}, O_{2}\right] / 2}$, is rigorously applicable if and only if $\left[O_{1},\left[O_{1}, O_{2}\right]\right]=\left[O_{2},\left[O_{1}, O_{2}\right]\right]=0$. This is not obviously the case for squared Fock operators. However, approximate expressions for $A_{i}{ }^{\mathrm{DC}}$ can still be derived due to the smallness of the interaction (i.e., $\beta<1$ ). At second order in $\beta$, the baby-universe interaction operator becomes

$$
A_{i}^{\mathrm{DC}} \simeq A_{i}^{(2)}=\beta\left(a_{i}^{\dagger 2}+a_{i}^{2}\right)-2 \beta^{2}\left(a_{i}^{\dagger} a_{i}+\frac{1}{4}\right) .
$$

In fact,

$$
\begin{aligned}
& e^{-\beta^{2} / 2}\left\langle 2 n_{2}\left|e^{\beta\left(a^{\dagger 2}+a^{2}-2 \beta a^{\dagger} a\right)}\right| 2 n_{1}\right\rangle \simeq e^{\beta^{2} / 2}\left\langle 2 n_{2}\left|e^{\beta a^{\dagger 2}} e^{\beta a^{2}}\right| 2 n_{1}\right\rangle, \\
& e^{\beta^{2} / 2} \sum_{r=0}^{\min \left(n_{1}, n_{2}\right)}\left\langle 2 n_{2}\left|\frac{\left(\beta a^{\dagger 2}\right)^{n_{2}-r}}{\left(n_{2}-r\right) !}\right| 2 r\right\rangle\left\langle 2 r\left|\frac{\left(\beta a^{2}\right)^{n_{1}-r}}{\left(n_{1}-r\right) !}\right| 2 n_{1}\right\rangle \\
&=(3.4),
\end{aligned}
$$

where we have assumed all the baby universes to be identical.

Equation (3.6) can be thought of as accounting for a nonlinear process by which, superimposed to independent baby-universe creation and annihilation in pairs, there is a mechanism which, starting from the initial threegeometry, first creates two baby universes, then destroys them to end at the final three-geometry. For the purposes of the present work, it will suffice nevertheless keeping things as simple as possible, so that we shall restrict to first order in $\beta$; i.e., we take for the baby-universe interaction operator the simple combination

$$
A_{i}^{\mathrm{DC}} \simeq A_{i}^{(1)}=\beta\left(a_{i}^{\dagger 2}+a_{i}^{2}\right),
$$

which accounts for the most elementary doubly connected wormhole interaction process by which baby universes are created and destroyed in pairs.

Thus, if $\phi$ denotes the background fields, the effective Lagrange density for quantum fields at low energy induced by interaction with doubly connected wormholes takes the form

$$
L \simeq L_{0}(\phi)+\sum_{i} L_{i}(\phi)\left(a_{i}^{\dagger 2}+a_{i}^{2}\right),
$$

where $L$ and $L_{0}$ are, as in the simply connected wormhole case, local functions of the background fields and their derivatives, and the $L_{i}$ 's are matter interaction Lagrangian terms which are also functions of the same arguments as for $L$ and $L_{0}$, but, contrary to the simply 
connected case, generally have a nonlocal character, as will be shown shortly. Here, the type of baby universe characterized by $i$ contains also the mutual position between the two baby universes forming a pair, on the inner boundary three-manifold; i.e., the Fock operators for baby universes are no longer independent of spacelike separations on the boundary three-manifold of a doubly connected wormhole. This is a consequence of the fact that correlated baby universes have a nonzero energy and momentum. It follows that, owing to the existing effective connection between these baby universes and the main spacetime, though such baby universes are no more at one location that at any other relative to the large region, their state will depend on their spacelike separation. Of course, it will be independent of the position of the center of mass of the involved baby-universe pair.

\section{TOPOLOGICAL BREAKDOWN OF LOCALITY}

Considering a simply connected wormhole topology is an unnecessary and restrictive simplification. Actually, an observer in the asymptotic region does not know anything about the number of components which are required to divide the four-manifold, so that if one wants to formulate the quantum state of wormholes by a path integral, one should include contributions from all possible wormhole topologies. Thus, the most general representation of the quantum state of a wormhole will be a density matrix rather than a wave function. I shall restrict myself here to the contribution from a doubly connected wormhole configuration. It is expected that multiply connected contributions will add quite more complication, but not new essentially different effects.

Following Coleman [9], we shall describe the theory in this section in terms of a Hamiltonian density in Minkowski space rather than a Lagrange density in Euclidean space. Then

$$
H=H_{0}(\phi)+\sum_{i} H_{i}^{I}(\phi) A_{i}^{\mathrm{DC}},
$$

where $H_{0}$ is a local function of the background scalar fields $\phi$ and their derivatives, while the $H_{i}^{I}$ 's form a linearly independent set of Hermitian functions depending on the same arguments as $H_{0}$, but having an essentially nonlocal character, as it will be shown in what fol-

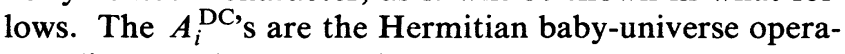
tors discussed in Sec. III, i.e.,

$$
A_{i}^{\mathrm{DC}}=a_{i}^{\dagger^{2}}+a_{i}^{2} .
$$

In Fock representation, a typical scale field operator $H_{i}^{I}(x)$, at spacelike position $x$, can be written in the form

$$
\begin{aligned}
H_{i}^{I}(x) & =\phi_{i}(x) \phi_{i}(x) \\
& =\int d \widetilde{P}\left(c^{2} e^{2 i p_{i} x}+c^{\dagger} c+c c^{\dagger}+c^{\dagger 2} e^{-2 i p_{i} x}\right),
\end{aligned}
$$

where the measure is typically given by $d \widetilde{\boldsymbol{P}}$ $=\left(d^{4} p /(2 \pi)^{3}\right) \delta\left(p^{2}-m^{2}\right) \theta\left(p_{0}\right)$. Here, $m$ denotes the inverse of the baby-universe characteristic proper radius, and $\theta\left(p_{0}\right)=1$ for $p_{0}>0$, and $\theta\left(p_{0}\right)=0$, otherwise.

The commutation relations for the fields are derived from a local Lagrange density [9]. Hence, prior to any interaction with wormholes which may topologically induce some modification of the requirement of locality, the fields involved should commute for spacelike separations. This property is obviously satisfied by the free-field Hamiltonian $H_{0}$, and is preserved by the interaction terms $H_{i}^{I}$ only for simply connected wormholes. The almost instantaneous interaction process can be regarded as the production of baby universes which take the scalar particles to the inner three-manifold away from the asymptotic regions. Thus, a simply-connected wormhole will preserve locality in the scalar field particle operators, as in that manifold one point on the flat region is mapped into one point on the inner three-manifold. The invariance of the Hamiltonian under spacelike location will therefore demand that if the fields commute for spacelike separation on the asymptotic region, then the operators $H_{i}^{I}$ will always commute for spacelike separations during interaction with simply connected wormholes. This is not guaranteed however for other wormhole topologies. If the wormhole is doubly connected, one wormhole end at $x_{0}$ will be topologically mapped into two spacelike separated points on the inner wormhole three-manifold. It follows that, although the locality demand on the asymptotic region will still imply commutativity of the operators $H_{0}$ for spacelike separations, it will not preserve the commutativity for the interaction operators $H_{i}^{I}$ for spacelike separations which exactly match the proper distance between the two disjoint three-manifold components on the inner wormhole boundary. For the doubly connected wormhole discussed in Sec. II, such a proper distance is $(2 K)^{1 / 2}$. For this case, we have then

$\sum_{i}\left[H_{i}^{I}(x), H_{i}^{I}(y)\right]=\sum_{i} D_{i}(x-y) \delta\left(2 K-(x-y)^{2}\right)$,

where the dependence of $D_{i}$ on $x-y$ follows from the requirement of invariance under spacelike translations, and we have introduced just one type $i$ of baby universe because of the identity of the two disjoint inner threemanifolds.

The $\delta$ function in (4.4) has been introduced to account for the additional requirement that if (given that the commutator ought to be invariant under changes of location on the large space) two scalar particles are at any relative proper distance other than $(2 K)^{1 / 2}$ on the inner wormhole three-manifold, then at least one of these particles will be disconnected from the whole manifold. This would obviously lead to a vanishing commutator. The $\delta$ function satisfies, moreover, the required invariance under spacelike translations as it depends only on relative distances.

We shall evaluate now $D_{i}$. We note that, except for their respective spacelike positions $x$ and $y$, the two members of a baby-universe pair are otherwise identical. Spacelike separation $x-y$ will fix furthermore all other distinguishing characteristics shared by the two baby universes in the pair. We can identify then the index $i$ with the spacelike separation between the two inner three-surfaces of the wormhole and, since $x_{0}=\frac{1}{2}(x-y)$ varies in a continuous fashion, the index $i$ becomes continuous as well. This implies that the summation on $i$ 
should be replaced by an integration over $x_{0}$ and that index $i$ can be dropped off from our formulas, i.e.,

$$
A_{i}^{\mathrm{DC}} \rightarrow A^{\mathrm{DC}}(x-y) \rightarrow A^{\mathrm{DC}}\left(x_{0}\right) .
$$

The part of (4.4) which is invariant under spacelike translations will be then proportional to

$i \int d^{4} x_{0} \int d \widetilde{P}\left(c^{\dagger} c+\frac{1}{2}\right) \sin [2 p(x-y)] \delta\left(2 K-(x-y)^{2}\right)$.

Taking then for our Lorentzian case $m=\left(R_{0}^{2}\right.$ $-K)^{-1 / 2},(x-y)^{2}<0$, we can perform the integrations over $p$ and $x_{0}$. We obtain finally

$$
\begin{aligned}
& \int d^{4} x_{0}\left[H^{I}(x), H^{I}(y)\right]=D_{0}\left(c^{\dagger} c+\frac{1}{2}\right) \sinh \left(\frac{8 K}{R_{0}^{2}-K}\right)^{1 / 2}, \\
& x_{0}=\frac{1}{2}(x-y)
\end{aligned}
$$

in which $D_{0}$ is a constant $c$ number of order unity. Note that this commutator will be zero only at the limit $K \rightarrow 0$, i.e., when the wormhole becomes simply connected.

The commutation relation $(4.7)$ can be generalized to the case in which the interaction operators $H^{I}$ are given in terms of the field operators $\phi$ as $H^{I} \propto \phi^{2 p}$ to read

$$
\int d^{4} x_{0}\left[H^{I}(x), H^{I}(y)\right]=D_{0}\left(c^{\dagger} c+\frac{1}{2}\right)^{p} \sinh \left(\frac{8 K}{R_{0}^{2}-K}\right)^{1 / 2} \text {. }
$$

Once we have obtained the commutator for the interaction Hamiltonian terms $H^{I}$ for spacelike separations, we shall consider the case of the total Hamiltonian density given by (4.1). The demand of Lorentz invariance of the perturbation theory will imply, according to Coleman [9], that the total Hamiltonian density $H$ must commute with itself for spacelike separations, and hence leads to an overall demand of locality. It follows then that

$$
\int d^{4} x_{0}\left[H^{I}(x) A_{x}^{\mathrm{DC}}\left(x_{0}\right), H^{I}(y) A_{y}^{\mathrm{DC}}\left(x_{0}\right)\right]=0,
$$

where the subscripts $x$ and $y$ for $A^{\mathrm{DC}}\left(x_{0}\right)$ do not mean explicit dependence on $x$ and $y$, but express the distinguishing feature for a single baby universe in the pair that the scalar fields interact just with one of the two disjoint components on the inner wormhole three-boundary. Nonlocality on that boundary is however preserved because the correlation between baby universes causes each inner disjoint three-surface to know exactly what is going on in the other.

Since the requirement of invariance under spacelike translations in our Fock spaces implies that all operators $H^{I}$ must commute with all operators $A^{\mathrm{DC}}$, from (4.9) it can be readily obtained that, for spacelike separations equal to $(2 K)^{1 / 2}$,

$$
\begin{aligned}
& H^{I}(y) H^{I}(x)\left[A_{x}^{\mathrm{DC}}\left(x_{0}\right), A_{y}^{\mathrm{DC}}\left(x_{0}\right)\right] \\
& =A_{x}^{\mathrm{DC}}\left(x_{0}\right) A_{y}^{\mathrm{DC}}\left(x_{0}\right)\left[H^{I}(y), H^{I}(x)\right] \neq 0,
\end{aligned}
$$

and, hence, for $(x-y)^{2}=-2 K$,

$$
\left[A_{x}^{\mathrm{DC}}\left(x_{0}\right), A_{y}^{\mathrm{DC}}\left(x_{0}\right)\right] \neq 0 \text {. }
$$

We see then that, whenever the interaction Hamiltonian densities are nonlinear in the creation and annihilation operators for baby universes [see Eqs. (4.1) and (4.2)], the interaction operator for baby universes does not commute with itself for nonzero spacelike separations equal to $(2 K)^{1 / 2}$. This result will become crucial to induce a breakdown of quantum coherence in the quantum fields at low energy of the asymptotic region, and is connected to the feature that the fundamental process which is taking place creates or annihilates baby universes two at a time.

We shall simply sketch now the main arguments and conclusions that follows form (4.8) and (4.11). They can be readily understood when confronted to the detailed analysis carried out by Coleman to get just the opposite

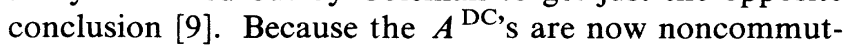
ing Hermitian operators, and

$$
\int d x_{0}^{4}\left[H, A^{\mathrm{DC}}\right] \neq 0, \quad \int d x_{0}^{4}\left[O, A^{\mathrm{DC}}\right] \neq 0,
$$

(where $O$ denotes any local operator) the space of the baby-universe states will be different for every particular operator $A_{x}^{\mathrm{DC}}\left(x_{0}\right)$, and any state in the state space of $A_{x}^{\mathrm{DC}}\left(x_{0}\right)$ will not remain in the same state space under time evolution, or under the action of a local operator. This ultimately leads to an unavoidable loss of quantum coherence which is induced by the fact that the baby universes are produced or destroyed in pairs. The full argument can be straightforwardly generalized to any multiply connected wormhole configuration. Thus, since such configurations should all contribute the full wormhole quantum state, we may conclude that quantum gravity induces a breakdown of predictability in the observable quantum-field sector at low energy of physical theories. In particular, if, in the light of the above ideas, we analyze the process of doing $N$ separated identical experiments discussed by Coleman [9], we obtain an outgoing density matrix which is just the product of $N$ oneexperiment density matrices.

\section{WORMHOLES AND QUANTUM MECHANICS}

The main result in this work is that, if we allow contributions from nonsimply connected wormholes to the topology of spacetime, there will be an irretrievable loss of quantum coherence in any physical theory aimed at describing the evolution of quantum fields at low energy. However, the loss of quantum coherence induced by simply connected wormholes which was advocated by Hawking does not take place. It is also obtained that the quantum state of wormholes as defined in terms of a path integral must necessarily contain contributions from multiply connected wormholes configurations; i.e., it ought to be given as a density matrix rather than a wave function.

The reason why one has a loss of quantum coherence is that, though the demand of causal locality on the asymptotic region is preserved for quantum fields interacting with simply connected wormholes, it is not when the wormholes have multiply connected topology.

As was pointed out in the Introduction, the successive proposals for loss of quantum coherence prompted a lot of opposition, both when it was assumed to be induced by 
gravitational horizons and when it was after associated with wormholes. There seems to be some feeling that the gravitationally induced loss of quantum coherence will demolish the predictability still surviving in conventional quantum mechanics which can be expressed by the unitary evolution of the state vector according to the Schrödinger equation. However, by simply requiring some objective character for the state vector (meaning that in the two-slits experiment, for instance, the objective reality of the system actually consists of the particle being partly at one slit and partly at the other slit, both together in some complex linear combination), the statevector reduction commonly assumed to occur at quantum measurement will actually imply a loss of the quantum coherence of the system. Thus, it could well be that a similar effect induced by wormholes (which we are so afraid of) was already implicitly required by conventional quantum mechanics as the missing essential ingredient to explain the collapse of the wave function during measurement. The price one should pay for reliving our fears this way would be by somehow accepting Einstein's view [17] that quantum mechanics is an incomplete theory, the missing piece being some form of quantum gravity.

Actually, inspired by the ideas put forward recently by Penrose [18], I am proposing here that the complete quantum representation of any physical system is given by a functional $\Omega\left(q_{m}, q_{w}\right)$, which in addition to the usual quantum-mechanical variables $q_{m}$, depends on some relevant wormhole variables $q_{w}$. Such a functional would not be generally factorizable as a product $\Omega\left(q_{m}\right) \Omega\left(q_{w}\right)$ because of the existing interaction between the two subsystems represented by the terms $H^{I} A$ discussed in this work.

This proposal can be motivated as follows. (i) The unitary evolution of the state vector is deterministic and preserves the linearity of quantum mechanics. The state vector remains time symmetric during unitary evolution. The gravitational sector for the state vector during unitary evolution must correspond to simply connected wormholes which are describable by a wave functional and induced no loss of quantum coherence in the state vector as they originate from a linear dynamics. These wormholes preserve furthermore, local causality and time symmetry, two characteristics which are required in the unitary evolution of the matter state vector. The unob- servability of this state would follow, moreover, from the unobservable character of the wave function of wormholes. (ii) The nonlinear quantum-mechanical evolution corresponding to the reduction of the state vector of matter required an associated wormhole state sector able to induce the necessary loss of quantum coherence. An observer would be aware of this loss through measurement, and the reduction process should be nonlocal, time asymmetric [18], and expressed in terms of real probabilities rather than probability amplitudes. All of these required characteristics are actually expected to be induced by the wormhole state sector if this is given in terms of an observable density matrix (note that the violation of local causality actually implies time asymmetry and must be induced by some nonlinear effect, probably of the kind discussed in Sec. II).

Thus, at least apparently, everything seems to fit very comfortably in this proposal. The main question arises nevertheless from the above picture: What is the cause by which one should suddenly replace simply connected wormholes by multiply connected wormholes when some measurement is being carried out on the system? Although I do not know the answer, at this point it is tempting to look at Wigner's view [19] where unitary evolution holds until consciousness gets involved in the physical system. Actually, only with the entry of the result of the measurement into somebody's consciousness will the entire pyramid of successive quantum states advocated by the Copenhagen school collapse into concrete reality [20]. Using the language of this paper, one would say that simply-connected wormholes should be the only topological fluctuations which may interact with matter when only inanimate objects without consciousness can influence the physical system, while multiply connected wormholes have to be regarded as something related to the distinguishing characteristics of a living being provided with consciousness. Of course, much work and thought remain to be done in this direction.

\section{ACKNOWLEDGMENTS}

This work was supported by an Accion Especial of the Consejo Superior de Investigaciones Científicas (CSIC). It is a pleasure to thank M. Santos, C. Siguenza, and L. J. Garay for useful discussions.
[1] S. W. Hawking, Phys. Rev. D 14, 2460 (1976); in Quantum Gravity 2, Proceedings of the Oxford Conference, Oxford, England, 1980, edited by C. J. Isham, R. Penrose, and D. W. Sciama (Clarendon, Oxford, 1981); Commun. Math. Phys. 87, 395 (1982).

[2] S. W. Hawking, D. N. Page, and C. N. Pope, Nucl. Phys. B170, 283 (1980); D. N. Page, Phys. Rev. Lett. 44, 301 (1980); Gen. Relativ. Gravit. 14, 299 (1982); A. Strominger, Phys. Rev. Lett. 52, 1733 (1984); T. Banks, M. Peskin, and L. Susskind, Nucl. Phys. B244, 125 (1984); J. Ellis, J. Hagelin, D. N. Nanopoulos, and M. Srednicki, ibid. B241, 381 (1984).

[3] D. J. Gross, Nucl. Phys. B236, 349 (1984).
[4] S. W. Hawking, Phys. Lett. B 195, 337 (1987); Phys. Rev. D 37, 904 (1988).

[5] S. B. Giddings and A. Strominger, Nucl. Phys. B306, 890 (1988).

[6] G. V. Lavrelashvili, V. A. Rubakov, and P. G. Tyniakov, Pis'ma Zh. Eksp. Teor. Fiz. 46, 134 (1987) [JETP Lett. 46, 167 (1987)]; Nucl. Phys. B299, 757 (1988).

[7] S. W. Hawking, Mod. Phys. Lett. A 5, 145 (1990); 5, 464 (1990).

[8] P. F. González-Díaz, Nucl. Phys. B351, 767 (1991).

[9] S. Coleman, Nucl. Phys. B307, 867 (1988); S. B. Giddings and A. Strominger, ibid. B307, 854 (1988).

[10] P. F. González-Díaz, Phys. Rev. D 42, 3983 (1990). 
[11] S. W. Hawking and R. Laflamme, Phys. Lett. B 209, 39 (1988).

[12] W. G. Unruh, Phys. Rev. D 40, 1053 (1989).

[13] G. W. Gibbons, S. W. Hawking, and M. J. Perry, Nucl. Phys. B138, 141 (1978).

[14] P. F. González-Díaz, Phys. Lett. B 233, 85 (1989).

[15] J. J. Halliwell and R. Laflamme, Class. Quantum Grav. 6, 1839 (1989).

[16] P. F. González-Díaz, Instituto de Optica Report No. QGC-02-91, 1991 (unpublished)

[17] A. Einstein, B. Podolsky, and N. Rosen, Phys. Rev. 47, 777 (1935).
[18] R. Penrose, in Quantum Gravity 2 [1]; in 300 Years of Gravitation, edited by S. W. Hawking and W. Israel (Cambridge University Press, Cambridge, England, 1987); in Quantum Concepts in Space and Time, Proceedings of the Oxford Conference, Oxford, England, 1984, edited by R. Penrose and C. J. Isham (Clarendon, Oxford, 1986).

[19] E. P. Wigner, in The Scientist Speculates, edited by I. J. Good (Heinemann, London, 1961); Am. J. Phys. 31, 6 (1963).

[20] P. C. W. Davies, in The Ghost in the Atom, edited by P. C. W. Davies and J. R. Brown (Cambridge University Press, Cambridge, England, 1986). 